\title{
Neogenin, a regulator of adult hippocampal neurogenesis, prevents depressive-like behavior
}

Dong Sun $\mathbb{1}^{1,2}$, Xiang-Dong Sun², Lu Zhao ${ }^{1,2}$, Dae-Hoon Lee², Jin-Xia Hu 2,3 , Fu-Lei Tang ${ }^{2}$, Jin-Xiu Pan², Lin Mei², Xiao-Juan Zhu' ${ }^{1}$ and Wen-Cheng Xiong ${ }^{2}$

\begin{abstract}
Adult neurogenesis in hippocampal dentate gyrus (DG) is a complex, but precisely controlled process. Dysregulation of this event contributes to multiple neurological disorders, including major depression. Thus, it is of considerable interest to investigate how adult hippocampal neurogenesis is regulated. Here, we present evidence for neogenin, a multifunctional transmembrane receptor, to regulate adult mouse hippocampal neurogenesis. Loss of neogenin in adult neural stem cells (NSCs) or neural progenitor cells (NPCs) impaired NSCs/NPCs proliferation and neurogenesis, whereas increased their astrocytic differentiation. Mechanistic studies revealed a role for neogenin to positively regulate Gli1, a crucial downstream transcriptional factor of sonic hedgehog, and expression of Gli1 into neogenin depleted NSCS/NPCs restores their proliferation. Further morphological and functional studies showed additional abnormities, including reduced dendritic branches and spines, and impaired glutamatergic neuro-transmission, in neogenin-depleted new-born DG neurons; and mice with depletion of neogenin in NSCs/NPCs exhibited depressivelike behavior. These results thus demonstrate unrecognized functions of neogenin in adult hippocampal NSCs/NPCspromoting NSCs/NPCs proliferation and neurogenesis and preventing astrogliogenesis and depressive-like behavior, and suggest neogenin regulation of Gli1 signaling as a possible underlying mechanism.
\end{abstract}

\section{Introduction}

In adult mammalian brains, two neurogenic regions retain the ability to generate new neurons ${ }^{1}$. First, it is the subventricular zone (SVZ) of the lateral ventricular region, which generates neuroblasts that migrate into olfactory bulb $(\mathrm{OB})$ through the rostral migratory stream and then differentiate into GABA and dopamineproducing interneurons ${ }^{2,3}$. Second, it is the subgranular zone of dental gyrus (DG) of hippocampus, which gives

\footnotetext{
Correspondence: X-J. Zhu (zhuxj720@nenu.edu.cn) or W-C. Xiong (WXIONG@augusta.edu)

${ }^{1}$ Key Laboratory of Molecular Epigenetics of Ministry of Education, Institute of Cytology and Genetics, Northeast Normal University, Changchun, Jilin 130024, China

${ }^{2}$ Department of Neuroscience \& Regenerative Medicine and Department of Neurology, Augusta University, Augusta, GA 30912, USA

${ }^{3}$ Department of Neurology, The affiliated hospital of Xuzhou Medical

University, Xuzhou, Jiangsu Province 221002, China

Edited by M. Campanella
}

rise to progenitors that undergo several developmental stages defined by specific markers and finally become local excitatory granule neurons ${ }^{4,5}$. Mature granule neurons can integrate into local circuitry and perform corresponding functions ${ }^{6}$. Reduction of adult neurogenesis impairs synaptic transmission and plasticity in the DG, and both long-term potentiation and long-term depression are regulated by adult neurogenesis ${ }^{7}$. Furthermore, several studies demonstrate that defective adult hippocampal neurogenesis contributes to depression ${ }^{8,9}$.

Adult neurogenesis is tightly controlled by cell intrinsic factors as well as local microenvironment, including transcription factors, growth factors, neuropeptides, and neurotransmitters. Among the intrinsic factors, Tlx, Sox2 and miR-124 are well known in regulating the proliferation of neural stem cells/neural progenitor cells (NSCs/ $\mathrm{NPCs})^{10-12}$. In addition, sonic hedgehog (Shh), Wnt $/ \beta$ - 
catenin, and Notch gene homolog2 (Notch2) pathways have been studied extensively for their functions in maintenance and self-renewal of NSCs/NPCs ${ }^{13-15}$. We are all aware that the SGZ is enriched in exuberant neuronal communications between mature granule neurons and inhibitory interneurons ${ }^{16}$, major neurotransmitters like Glutamate and GABA have long been implicated in regulating adult hippocampal neurogenesis ${ }^{17,18}$. Recently, mature granule neurons were also thought to compete with new-born neurons for synaptic inputs, and further affected NSCs activation ${ }^{19}$.

Neogenin, a member of deleted in colorectal cancerfamily transmembrane proteins, appears to be a receptor or co-receptor for multi-ligands, including netrins, repulsive guidance molecules, and bone morphogenetic proteins (BMPs) ${ }^{20-22}$. Thus, it is implicated in various cellular functions in the brain, such as axon guidance, neuronal differentiation, neural tube formation, neuronal survival $^{21,23-25}$, adult SVZ-OB neurogenesis ${ }^{26}$, and olfactory epithelium development ${ }^{27}$. In addition to the brain, neogenin plays important roles in non-brain organs, such as endochondral bone formation ${ }^{28}$, digit patterning ${ }^{29}$, and ion hemostasis ${ }^{30,31}$. Notice that neogenin is highly expressed in NSCs/NPCs, however, its function in adult hippocampal neurogenesis remains largely unknown.

Here, we provide evidence for neogenin's function in regulation of adult mouse hippocampal neurogenesis as well as depressive-like behavior. Neogenin is highly expressed in NSCs/NPCs and immature neurons. Selective depletion of neogenin in hippocampal NSCs/NPCs impaired their proliferation and neurogenesis, but increased their astrocytic differentiation. Further analysis showed that neogenin-deficient new-born neurons in DG displayed multiple abnormities, including reduced dendritic branches and spines and impaired glutamatergic neuro-transmission. In addition, mice with depletion of neogenin in NSCs/NPCs showed depressive-like behavior, supporting the view for adult hippocampal neurogenesis to be crucial in hippocampus-dependent mood regulation. Together, these results demonstrate critical functions of neogenin in promoting adult hippocampal neurogenesis and preventing depressive-like behavior.

\section{Results}

Neogenin expression in NSCs/NPCs and immature neurons in adult mouse hippocampal DG

To investigate neogenin's function in adult hippocampus, we first examined its expression by taking advantage of $L a c Z$ reporter in neogenin mutant mice. The $L a c Z$ gene is knocked in the intron of neogenin gene, thus, the Lac $Z$ activity, under the control of neogenin promoter, has been used as a reporter for neogenin's expression $^{28,31-33}$. The LacZ enzymatic activity by X-gal staining was strong in regions of subgranule zone (SGZ) of dentate gyrus (DG) and CA3, but weak in CA1 and CA2 zones (Fig. 1a and a'). The high level of LacZ expression in SGZ was further confirmed by immunostaining analysis using anti- $\beta$-gal antibody (Fig. $1 \mathrm{~b}$ and $\mathrm{b}^{\prime}$ ). As SGZ region contains various types of cells, including $\mathrm{Nestin}^{+}$or $\mathrm{GFAP}^{+}$radial glia-like NSCs, $\mathrm{Mcm}^{+}$nonradial NSCs (or transient amplifying NSCs/NPCs), glial cells (e.g., astrocytes and microglia), and $\mathrm{DCX}^{+}$immature neurons (Fig. 1c), we asked in which cell type(s) in the SGZ that neogenin is expressed. Co-immunostaining analysis showed that neogenin's $\beta$-gal reporter was detected in $\mathrm{Nestin}^{+}$or $\mathrm{GFAP}^{+}$radial glia-like NSCs (Fig. 1d, e), but enriched in $\mathrm{Mcm}^{+}$transient amplifying NSCs/NPCs, as well as DCX ${ }^{+}$immature neurons (Fig. 1f, g). The $\beta$-gal signal was barely detected in the granule cell layer (GCL) where $\mathrm{NeuN}^{+}$mature neurons reside (Fig. 1h), and un-detectable in microglia (data not shown). These results thus demonstrate abundant neogenin's expression in NSCs/NPCs and immature neurons in the SGZ (Fig. 1c), implicating neogenin's function in adult hippocampal neurogenesis.

\section{Impaired adult hippocampal neurogenesis in mice that selectively knocked out neogenin in NSCs/NPCs}

To investigate neogenin's function in NSCs/NPCs, we

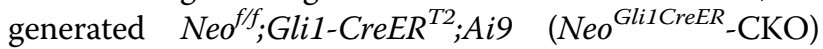
mice by crossing floxed neogenin $\left(\mathrm{Neo}^{\mathrm{fff}}\right)$ with Gli1$\mathrm{CreER}^{T 2}$;Ai9 mice (Supplementary Figs. S1A, B, S2A, B) for the following reasons. First, neogenin's expression in SGZ resembles to that of Gli1 (GLI-Kruppel family member), a transcription factor downstream of Shh signaling that has been used as a sensitive readout of Shh activity to label Shh-responding cells in adult hippocampus $^{34}$. Second, the expression of Cre in SGZ of Gli1$C r e E R^{T 2}$ mice (inducible Cre recombinase expression driven by Gli1 promotor) was verified by crossing $A i 9$ mice with Gli1-CreER ${ }^{T 2}$ to get Gli1-CreER ${ }^{T 2} ; A i 9$ mice (Supplementary Fig. S1A, B). Upon tamoxifen injection, Gli1-CreER ${ }^{T 2}$ is activated to mediate efficient recombination in Ai9, and the tdTomato is thus turned on to represent Shh-responding NSCs and their progenies (Supplementary Fig. S1C). As reported ${ }^{34-36}$, Nestin $^{+}$or $\mathrm{GFAP}^{+}$radial glia-like NSCs were positive for tdTomato in Gli1-CreER ${ }^{T 2} ; A i 9$ mice treated with tamoxifen for 7 days (D) (Supplementary Fig. S1D, E). The tdTomato was also detected in $\mathrm{Mcm}^{+}$transient amplifying NSCs/ NPCs and in $\mathrm{DCX}^{+}$immature neurons (Supplementary Fig. S1F, G). These results confirmed the expression of Gli1-Cre in NSCs/NPCs and immature neurons in the SGZ (Supplementary Fig. S1H), demonstrating a similar expression pattern of Gli1-Cre to that of neogenin. Finally, the endogenous neogenin was efficiently deleted in DG of $\mathrm{Neo}^{\text {GliICreER}}$-CKO mice, compared to that of control mice (Supplementary Fig. S2C-E). 


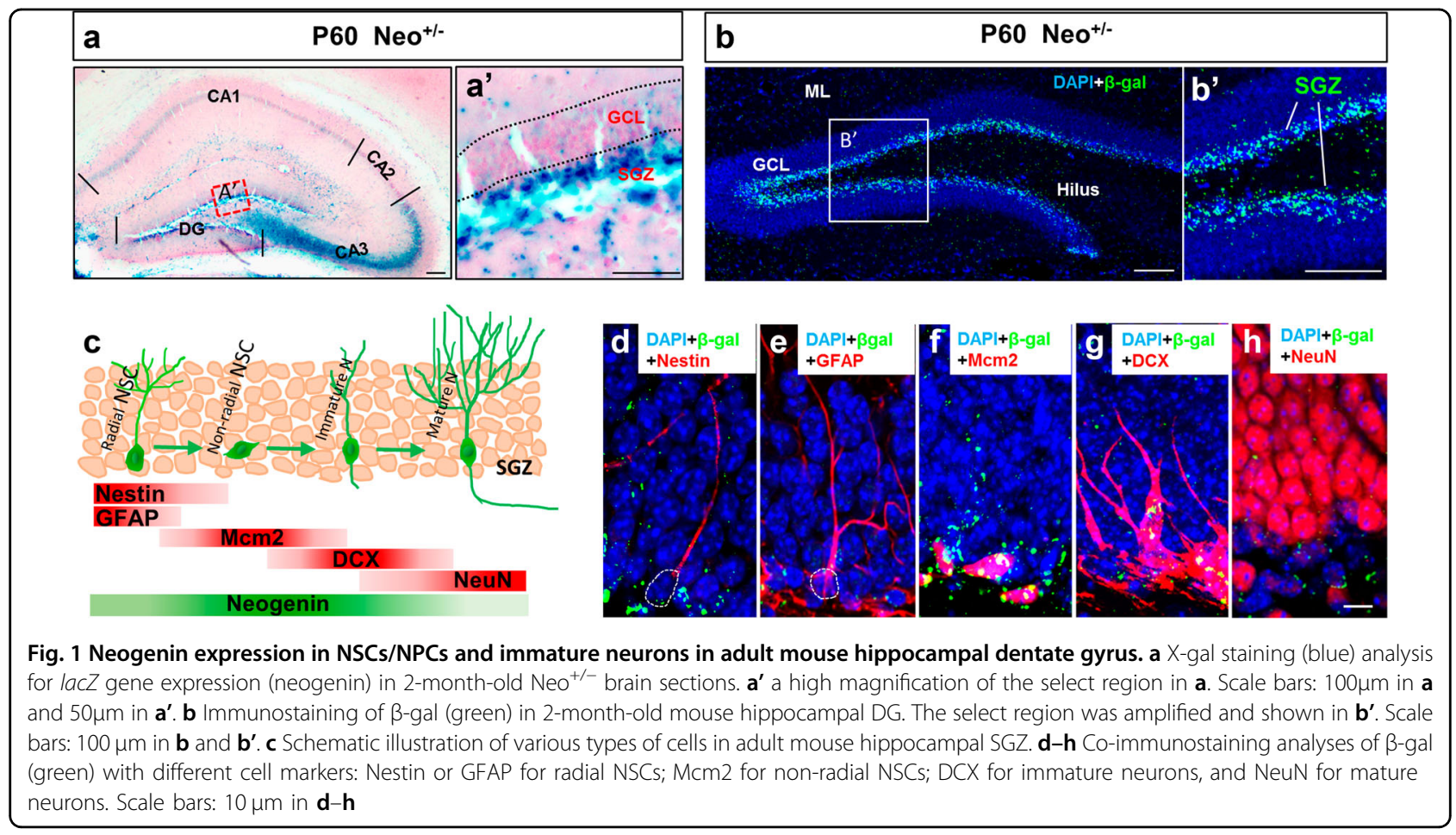

We next investigated neogenin's function in SGZ cells by comparing the numbers of $\mathrm{tdTomato}^{+}$cells between control $\left(\mathrm{NeO}^{+/+} ; \mathrm{Gli1}_{-} \mathrm{CreER}^{\mathrm{T2}} ; \mathrm{Ai} 9\right)$ and $\mathrm{NeO}^{\text {GlilCreER}_{-} \mathrm{CKO}}$ mice injected with tamoxifen at age of P60 (postnatal day 60 ) for 7, 14, 21 and 28-days, which are critical developmental stages for adult new-born neurons development (Fig. 2a). Whereas control mice showed time-dependent increases of tdTomato $^{+}$cells, $\mathrm{Neo}^{\text {GliICreER }_{-} \text {CKO mice }}$ displayed marked decreases in 14, 21, 28 days, but not 7 days, after tamoxifen injection (Fig. 2b, c). These results implicate neogenin in neurogenesis.

We then asked whether $\mathrm{DCX}^{+}$immature neurons were reduced in $\mathrm{NeO}^{\text {GliICreER }_{-} \mathrm{CKO}}$ mice. Both control and mutant mice were injected with tamoxifen at age of P60 for four times $(125 \mathrm{mg} / \mathrm{kg} / \mathrm{time}, 1 \mathrm{time} / 12 \mathrm{~h})$ to achieve higher efficiency, and 14 days after tamoxifen treatment, $\mathrm{DCX}^{+}$immature neurons were examined by immunostaining and Western blot analyses (Fig. 2d-h). Consistent with the result of decreased tdTomato ${ }^{+}$cells, $\mathrm{DCX}^{+}$ immature neuronal density was significantly lower in $\mathrm{NeO}^{\text {Gli1CreER}}$-CKO mice than that of control mice (Fig. 2d, e). In supporting this view, the DCX protein levels were reduced in $\mathrm{Neo}^{\text {Gli1CreER }}$-CKO hippocampus (Fig. 2g, h). The reduction in tdTomato ${ }^{+}$or $\mathrm{DCX}^{+}$cells was not due to an increased apoptosis, as cleaved-caspase3 (c-caspase3), a marker for apoptosis, was not increased in the mutant DG (Supplementary Fig. S3). Further analysis of $\mathrm{DCX}^{+}$neuronal morphology showed a much shorter dendritic process in the mutant DG (Fig. 2d, f), suggesting that in addition to the genesis of these immature neurons, neogenin also regulates their dendritic growth.

In addition to NSCs/NPCs in SGZ, neogenin is expressed in CA3 neurons and $\mathrm{GFAP}^{+}$astrocytes (Fig. 1 and data not shown). We thus asked whether neogenin expression in these cells plays a role in adult hippocampal neurogenesis. To this end, Neo ${ }^{\text {NestinCreER }}{ }_{-}$CKO, $\mathrm{NeO}^{\text {GFAPCreER}}{ }_{-} \mathrm{CKO}$, and $\mathrm{NeO}^{\text {Nex }}{ }_{-} \mathrm{CKO}$ mice were generated by crossing Neo ${ }^{f / f}$ with Nestin-CreER, GFAP-CreER, and $\mathrm{Nex}$-Cre mice, respectively. Injections with tamoxifen in

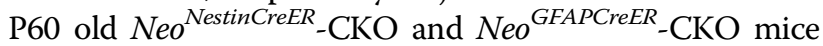
for 14 days (Supplementary Fig. S4A) resulted in selective knocking out of neogenin in NSCs and astrocytes, respectively (data not shown). $\mathrm{Neo}^{\mathrm{Nex}}$-CKO selectively knocked out neogenin in pyramidal neurons ${ }^{32}$. As shown in Supplementary Fig. S4B-D, DCX ${ }^{+}$immature neurons and their processes were markedly reduced in $\mathrm{Neo}^{\mathrm{NestinC}-}$ ${ }_{r e E R}$-CKO mice, compared with that of control mice, in agreement with the results in $\mathrm{NeO}^{\text {GliICreER}_{-}}$CKO mice. Interestingly, the reduction of $\mathrm{DCX}^{+}$immature neurons was not observed in Neo ${ }^{\text {GFAPCreER }}$-CKO (Supplementary Fig. S4E-G), nor $\mathrm{Neo}^{\mathrm{Nex}}$-CKO mice (Supplementary Fig. S4H-J), compared to their control mice. In contrast, an increase in $\mathrm{DCX}^{+}$neurons was detected in $\mathrm{Neo}^{\text {GFAPCreER }}$ CKO mice (Supplementary Fig. S4E-G). These results suggest that neogenin expression in NSCs/NPCs, but not astrocytes or neurons, promotes genesis of $\mathrm{DCX}^{+}$neurons. 


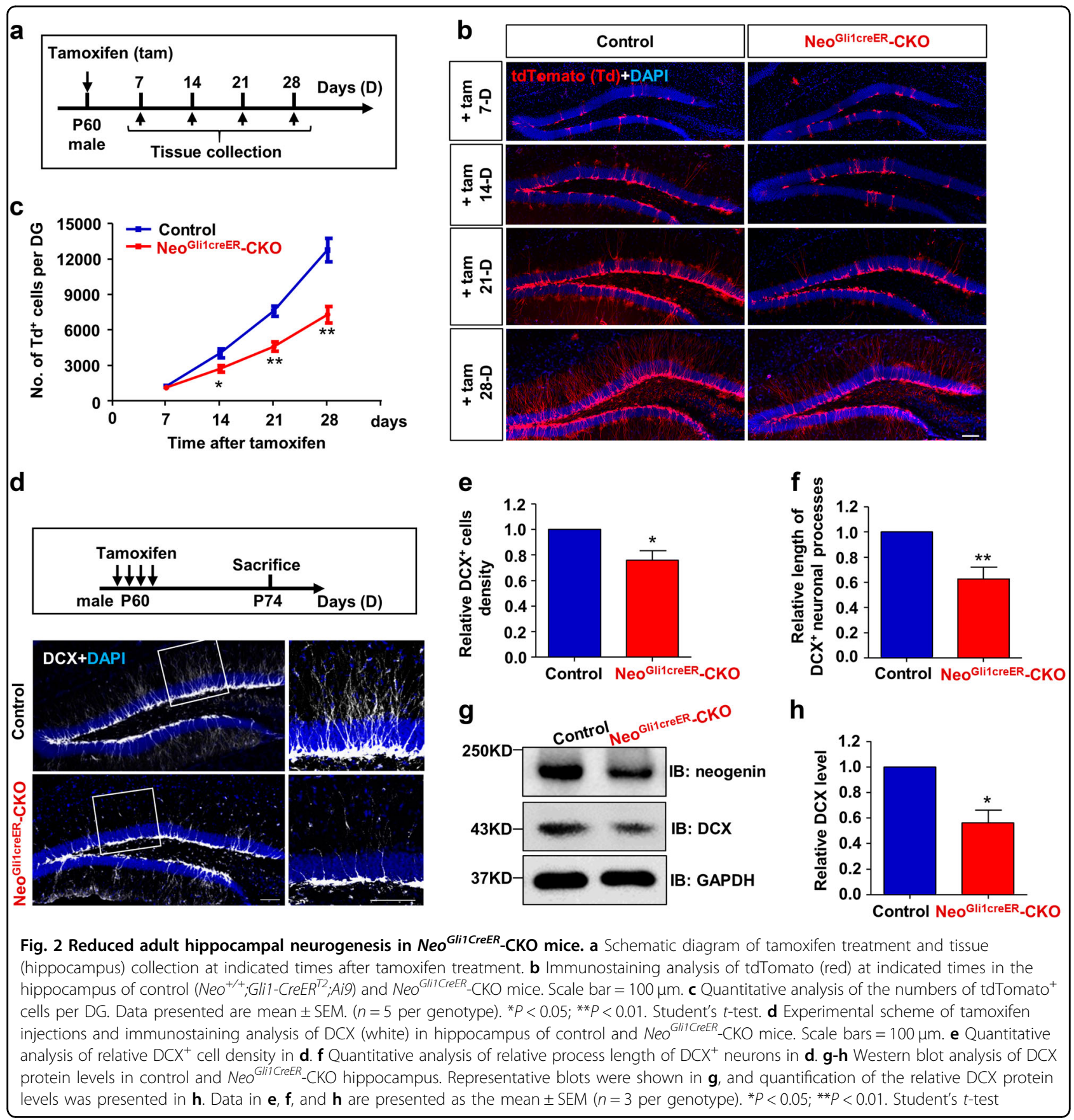

\section{Decreased proliferation, but increased astrocytic} differentiation, in neogenin-deficient NSCs/NPCs

$\mathrm{DCX}^{+}$immature neurons are derived from transient amplifying NSCs/NPCs ${ }^{37}$. These NSCs/NPCs, marked by $\mathrm{Mcm} 2$, are proliferative, and from nestin ${ }^{+}$or $\mathrm{GFAP}^{+}$ radial glia-like $\mathrm{NSCs}^{38}$ (Fig. 3a). To understand how neogenin in NSCs/NPCs promotes $\mathrm{DCX}^{+}$neurogenesis, we thus co-immunostained tdTomato with DCX, Mcm2, or GFAP antibodies in control and $\mathrm{Neo}^{\text {GliICreER}_{-} \text {CKO }}$ mice injected with tamoxifen at P60 for 14 days (Fig. 3b, d, f). To our surprise, the percentage of DCX and tdTomato double positive cells over total tdTomato ${ }^{+}$cells was

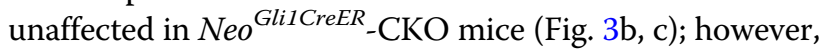
the percentage of $\mathrm{Mcm} 2$ and tdTomato double positive cells over total tdTomato ${ }^{+}$cells was significantly lower in the mutant mice than that of controls (Fig. 3d, e). In contrast, the percentage of GFAP and tdTomato double positive astrocytes over total tdTomato ${ }^{+}$cells was elevated in the mutant mice, but the $\mathrm{GFAP}^{+}$radial glia-like NSCs were unaffected (Fig. 3f-h). These results implicate 

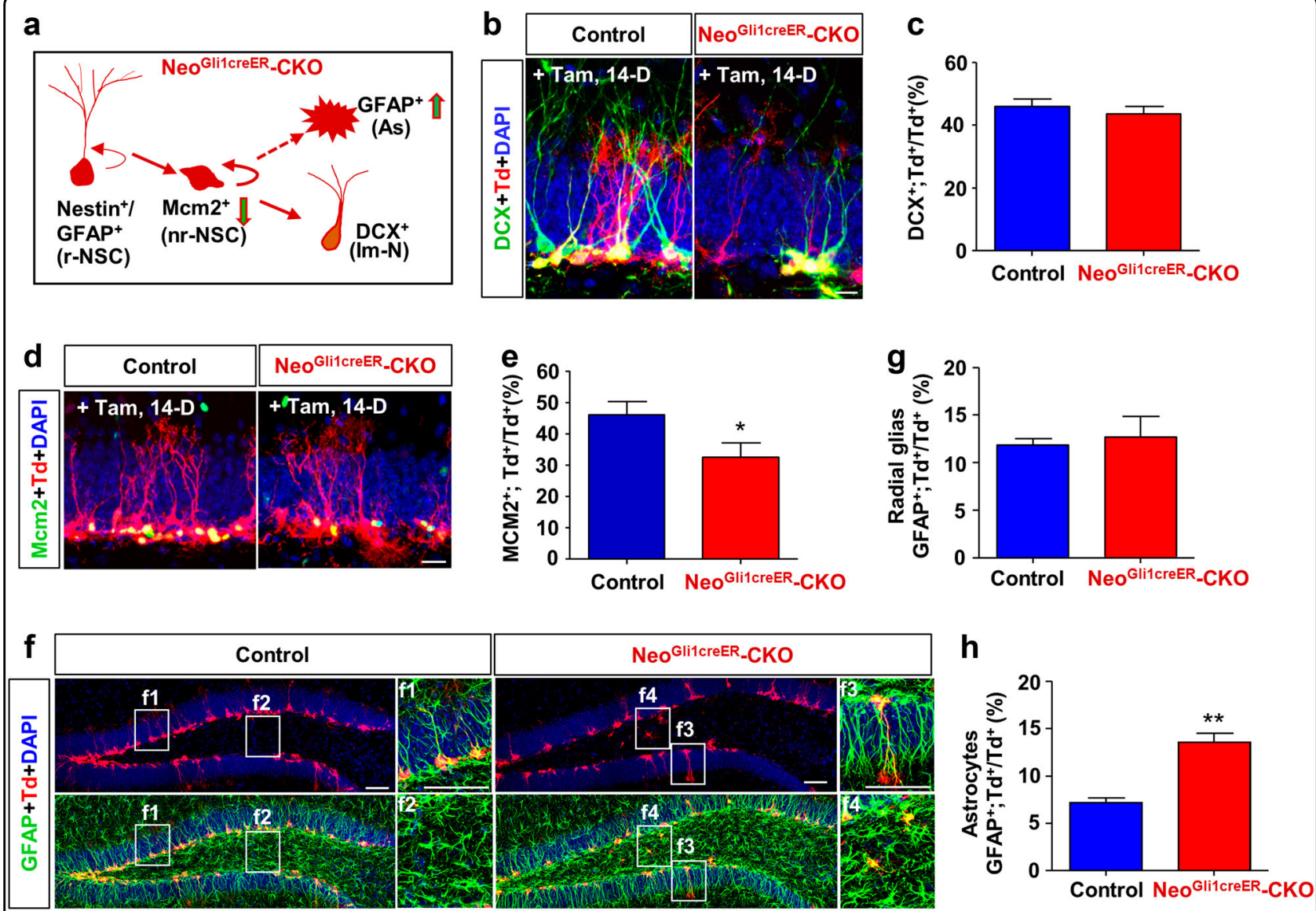

Fig. 3 Decreased proliferation, whereas increased astrocytic differentiation, and no effect of neuronal differentiation from neogenindeficient hippocampal NSCs/NPCs. a Model of the proliferation and differentiation of hippocampal NSCs/NPCs. b Double immunostaining of tdTomato (red) and DCX (green) in hippocampus of control and Neo GliicreER-CKO mice. Scale bars $=50 \mu \mathrm{m}$. c Quantification of the ratio of tdTomato ${ }^{+}$; $\mathrm{DCX}^{+}$over total tdTomato ${ }^{+}$cells. $\mathbf{d}$ Double immunostaining of tdTomato (red) and $\mathrm{Mcm} 2$ (green). Scale bars $=50 \mu \mathrm{m}$. e Quantification of the ratio of tdTomato ${ }^{+} \mathrm{Mcm}^{+}$over total tdTomato ${ }^{+}$cells. $\mathbf{f}$ Double immunostaining analysis of tdTomato (red) and GFAP (green). f1-f4 showed a higher magnification of the selected regions. Radial glia-like NSCs (f1 and f3): Bipolar shape with a long radial process, located in SGZ of DG. Astrocytes (f4): stellate shape with star-like short processes, located in SGZ, granule cell layer and hilus. Scale bars $=50 \mu \mathrm{m} . \mathbf{g}, \mathbf{h}$ Quantification of the radial glia-like NSCs $\mathbf{g}$ or astrocytes $\mathbf{h}$ ratio of tdTomato ${ }^{+}$; GFAP ${ }^{+}$over total tdTomato ${ }^{+}$cells. Data are presented as the mean \pm SEM. $\left(n \geq 500\right.$ cells). ${ }^{*} P<0.05 ;{ }^{* *} P<$ 0.01. Student's t-test

neogenin's dual functions in $\mathrm{Mcm}^{+}$NSCs/NPCs: a positive role for $\mathrm{Mcm}^{+} \mathrm{NSCs} / \mathrm{NPCs}$ proliferation and a negative role for astrogliogenesis.

To test this view, Brdu, a marker for cell proliferation, was injected into tamoxifen treated control and mutant mice, and $12 \mathrm{~h}$ after Brdu injection, mice were sacrificed for analysis (Fig. 4a). As shown in Fig. 4b, c, neogenin deletion resulted in a significant reduction of $\mathrm{Brdu}^{+}$cells in SGZ, supporting the view for a positive role of neogenin in NSC/NPC proliferation. This view was further supported by co-immunostaining analysis of Ki67 (an endogenous mitotic marker for cell proliferation), which showed decreased $\mathrm{Ki}^{6} 7^{+}$cells in $\mathrm{Neo}^{\mathrm{GlilCreER}_{-}}{ }_{\text {CKO mice, }}$ compared with that of control mice (Fig. 4d). Notice that the percentage of $\mathrm{Brdu}^{+}$and $\mathrm{Ki}^{-}$cells over total Brdu ${ }^{+}$ cells was higher in $\mathrm{Neo}^{\text {Gli1CreER}}$-CKO mice than that of controls (Fig. 4e), suggesting that more $\mathrm{Brdu}^{+} \mathrm{NSCs} /$ NPCs undergone cell cycle exit or had completed the cell cycle when neogenin was depleted, which supported the view for neogenin in promoting NSCs/NPCs proliferation, but suppressing their astrocytic differentiation (Fig. 4f).

To further examine neogenin's function in NSCs/NPCs proliferation, hippocampal NSCs were isolated from 2month old $\mathrm{Neo}^{f / f}$ and $\mathrm{Neo}^{\mathrm{Nestin}}$-CKO mice (Fig. 5a). As expected, endogenous neogenin was much lower in Neo ${ }^{\text {Nestin }}$-CKO NSCs than that of control NSCs (Supplementary Fig. S5A). The sizes of neurospheres were smaller in the mutant NSCs than those of controls, implicating a reduced growth of mutant NSCs (Supplementary Fig. S5B and $\mathrm{S} 5 \mathrm{C}$ ). We then planted NSCs on poly-L-ornithine and 
a

\begin{tabular}{|c|c|c|}
\hline $\begin{array}{c}\text { Tamoxifen } \\
\downarrow \downarrow \downarrow \downarrow\end{array}$ & $\begin{array}{l}\text { Brdu } \\
\downarrow t \downarrow t\end{array}$ & Sacrifice \\
\hline $\begin{array}{l}\mathrm{P} 60 \\
\text { male }\end{array}$ & P74 & 12-h post Brdu \\
\hline
\end{tabular}

C

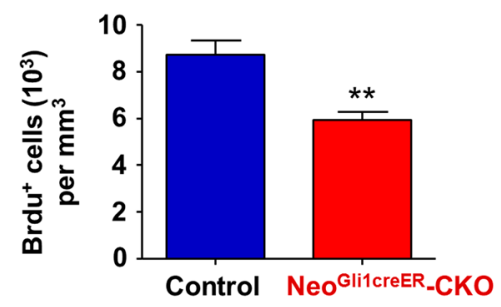

d

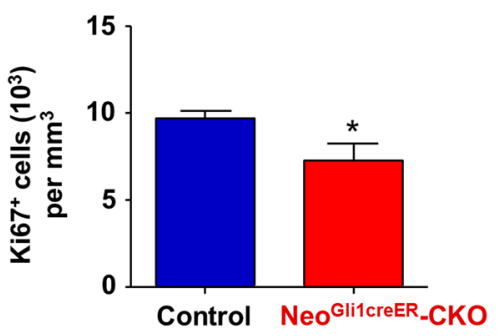

e

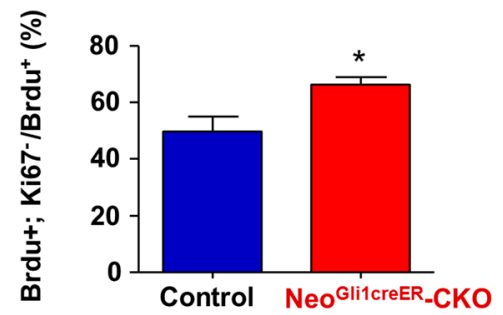

b
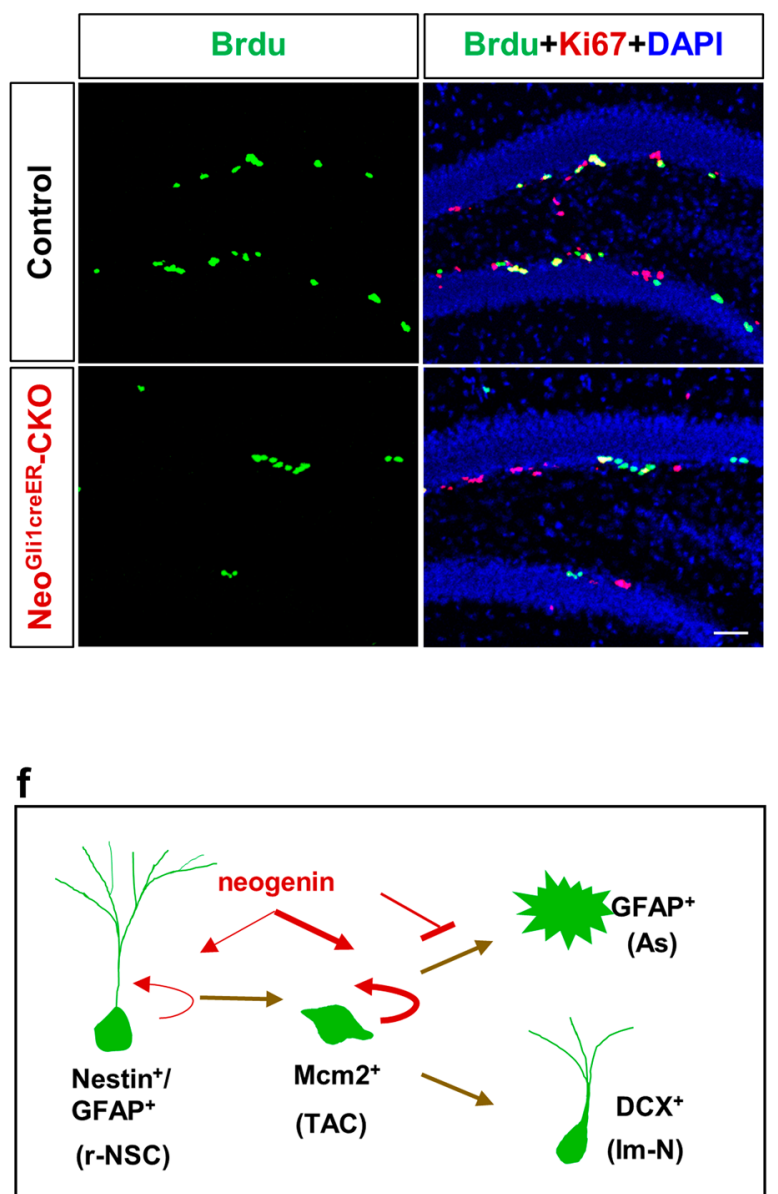

Fig. 4 Reduced proliferation, and promoted their cell cycle exit, from neogenin deficient hippocampal NSCs/NPCs. a Schematic diagram of multiple tamoxifen and Brdu injections for indicated time. $\mathbf{b}$ Double immunostaining of Brdu (green) and Ki67 (red) in hippocampus of control and Neo Glii CreER-CKO mice. Scale bars $=100 \mu \mathrm{m}$. c , d Quantification of Brdu ${ }^{+}$cell density in c and Ki67 ${ }^{+}$cell density in d. Data are mean \pm SEM. $(n=5$ per genotype). ${ }^{*} P<0.05$; ${ }^{*} P<0.01$. Student's $t$-test. e Quantification of the ratio of Brdu ${ }^{+} ; K_{i 67}$ over total Brdu ${ }^{+}$cells. Data are mean \pm SEM. ( $n \geq 500$ cells). ${ }^{*} P<0.05$. Student's $t$-test. $\mathbf{f}$ Model of neogenin promotion of NSC/NPC proliferation, but suppression of their astrocytic differentiation

laminin-coated coverslips, and cultured with proliferation medium (Neurobasal medium + B27 + GlutaMAX + FGF-2 + EGF) containing Brdu $(5 \mu \mathrm{M})$ for $6 \mathrm{~h}$. The Brdu incorporation in $\mathrm{Neo}^{\mathrm{Nestin}}$-CKO NSCs was markedly reduced, compared with that of controls (Fig. 5b, c). Taken together, these results provide in vitro and in vivo evidence for neogenin's function in promoting NSCs/ NPCs proliferation.

We next examined neogenin's function in astrocytic and neural differentiation from NSCs in vitro. The isolated NSCs from $\mathrm{Neo}^{f / f}$ and $\mathrm{Neo}^{\mathrm{Nestin}}$-CKO mice were planted onto poly-L-ornithine and laminin-coated coverslips and cultured with the differentiation medium (Neurobasal medium + B27 + GlutaMAX) for 3 days. Cells were fixed and immunostained with GFAP and DCX antibodies to label astrocytes and neurons, respectively (Fig. 5d, e). In line with the results in vivo, neogenin-depleted NSCs displayed increased $\mathrm{GFAP}^{+}$astrocytes, but a similar number of $\mathrm{DCX}^{+}$neurons, compared with those of control cells (Fig. 5f, g). These results thus provide additional evidence for neogenin's function in hippocampal NSCs to suppress astrocytic, but not neural, differentiation.

\section{Neogenin induction of Gli1 in hippocampal NSCs/NPCs to promote their proliferation}

Shh signaling pathway is known to be essential for sustaining the proliferation of NSCs/NPCs in hippocampus $^{13}$. We thus wonder whether neogenin regulates Shh pathway in hippocampal NSCs/NPCs to promote their proliferation. To test this view, we extracted mRNAs of hippocampal NSCs from $\mathrm{Neo}^{f / f}$ and $\mathrm{Neo}^{\mathrm{Nestin}}-\mathrm{CKO}$ mice. Real time-PCR analyses of genes in the Shh 


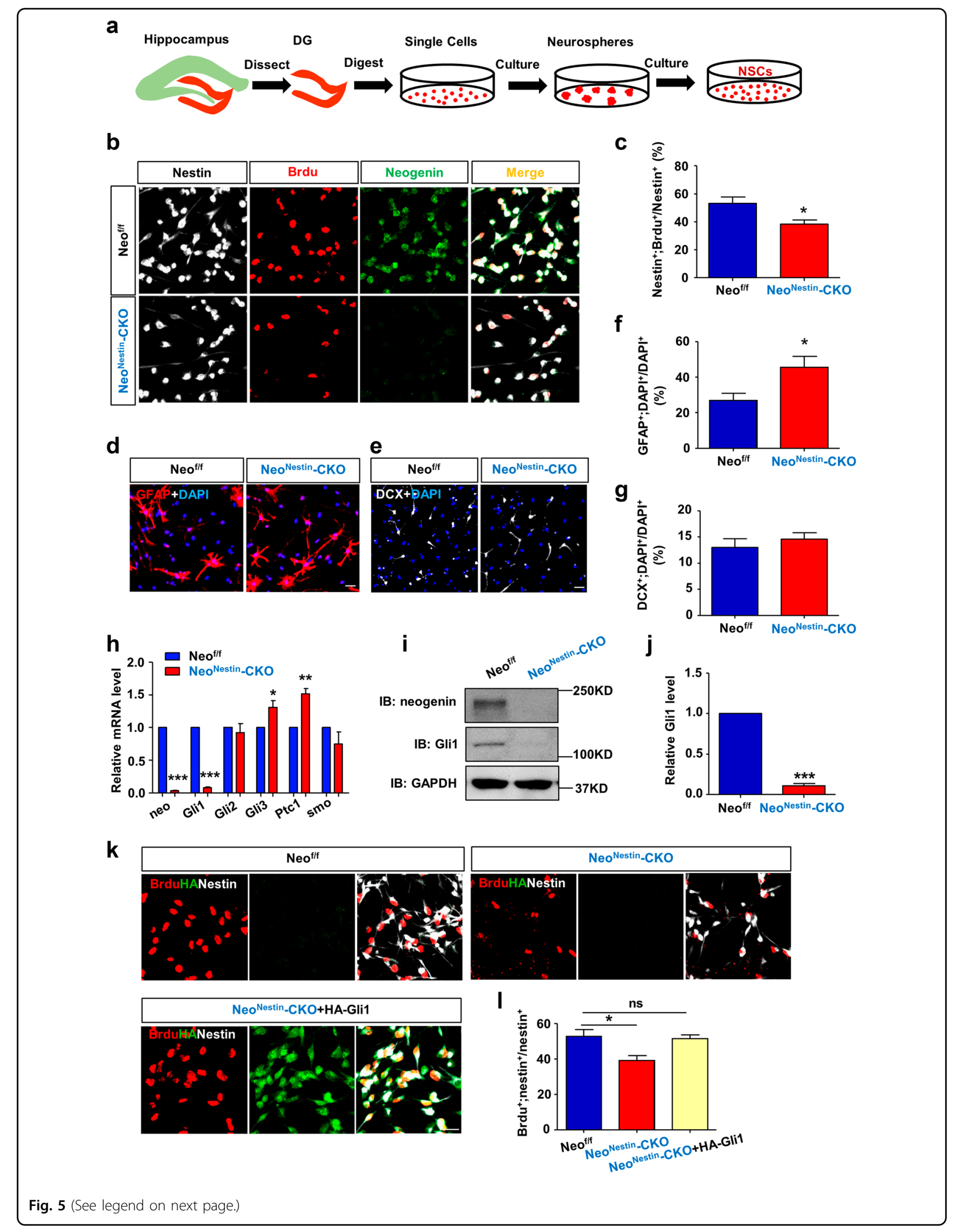


(see figure on previous page)

Fig. 5 Loss of neogenin reduced Gli1-mediated proliferation, but promoted astrocytic differentiation, in cultured NSCs. a Schematic drawing shows the isolation of DG-NSCs. b Brdu analysis for NSCs proliferation in vitro. Nestin (red), Brdu (white) and neogenin (green). ( $n \geq 1000$ cells from 5 slides). Scale bars $=50 \mu \mathrm{m}$. c Quantification of Brdu incorporated ratio of Brdu ${ }^{+} ; \mathrm{Nestin}^{+}$over total Nestin ${ }^{+}$cells. d, e Immunostaining of GFAP $\mathbf{d}$ or DCX $\mathbf{e}$ in differentiated cells in vitro. ( $n \geq 800$ cells from 5 slides). Scale bars $=20 \mu m$. $\mathbf{f}, \mathbf{g}$ Quantification of the ratio of GFAP $\mathbf{f}^{+}$or DCX $\mathbf{g}^{+} \mathbf{g}$ cells over total cells. $\mathbf{h}$ Real-time PCR (RT-PCR) analysis of relative gene levels of Shh signaling pathway (Normalized to NSCs of Neo f/f mice). i Western blot analysis of Gli1 protein levels in Neo ${ }^{f / f}$ and Neo ${ }^{\text {Nestin }}$-CKO NSCs. j Quantification of the relative Gli1 protein levels in I. ( $n=3$ per genotpye) $\mathbf{k}$ Brdu analysis for NSCs proliferation in three groups ( $\mathrm{NeO}^{\mathrm{f} / \mathrm{f}}$, Neo ${ }^{\text {Nestin }}-\mathrm{CKO}$ and Neo ${ }^{\text {Nestin }}-\mathrm{CKO}+\mathrm{Gli1}$ ). Scale bars $=10 \mu \mathrm{m}$. I Quantification of the Brdu incorporated ratio, $\mathrm{Brdu}^{+}$;Nestin ${ }^{+}$over total Nestin ${ }^{+}$cells. Data are presented as the mean \pm SEM. $\left(n \geq 1000\right.$ cells from 5 slides). ${ }^{*} P<0.05$; ${ }^{* *} P<0.01$; ${ }^{* * *}$ $P<0.001$. Student's $t$-test or one-way ANOVA plus post-hoc analysis

pathway, including Gli1-3, patched1 (Ptc1), and smoothen (smo), were examined. Intriguingly, Gli1, a transcriptional factor downstream of Shh pathway and a sensitive readout of Shh activity, was significantly lower in $\mathrm{NeO}^{\mathrm{Nestin}}{ }_{-} \mathrm{CKO}$ NSCs than that of $\mathrm{Neo}^{f / f}$ NSCs (Fig. 5h). In contrast, the mRNA levels of Gli3 and Ptc1 were increased in neogenin deficient NSCs (Fig. 5h). The reduction of Gli1 in Neo${ }^{\text {Nestin }}$-CKO NSCs was further confirmed by Western blot analysis (Fig. 5i, j). We then asked if the reduced Gli1 in neogenin deficient NSCs is responsible for the impaired proliferation. To this end, neogenin deficient NSCs infected with or without retroviruses expressing HAtagged Gli1 were cultured for 3-days, and incubated with the proliferation medium containing $5 \mu \mathrm{M}$ Brdu for $6 \mathrm{~h}$. Remarkably, expression of Gli1 in Neo ${ }^{\text {Nestin }}$-CKO NSCs showed a comparable level of Brdu incorporation to that of $\mathrm{Neo}^{\mathrm{f} / f} \mathrm{NSCs}$ (Fig. 5k, l), suggesting a critical role of Gli1 in mediating neogenin-promoted NSCs proliferation.

\section{Impaired dendritic morphogenesis and maturation in neogenin depleted adult new-born DG neurons}

Notice that in addition to the reduction of $\mathrm{DCX}^{+}$ neuronal density, their dendritic processes were much shorter in the mutant SGZ (Fig. 2d-f), implicating neogenin in promoting new born DG neuronal dendritic morphogenesis and maturation. We further tested this view by use of retrovirus driven Cre strategy, as retrovirus targets only dividing cells and labels their subsequent progenies $^{39}$. Retroviruses encoding Cre or d-Cre (an inactive Cre, as a control) with IRES-EGFP (under the control of CAG promoter) were injected into the DG of $\mathrm{Neo}^{f / f}$ mice to infect NSCs/NPCs, and 3 weeks later, GFPmarked DG neurons were examined (Fig. 6a). To accurately acquire the three-dimensional image, each final image was composed of ten serial $z$-plane images by Zeiss confocal system. Indeed, the dendritic complexity was considerably reduced in Cre expressing (neogenin-CKO) new born neurons, compared with that of controls assessed by sholl analysis (Fig. 6b, c). Neogenin deficient new born neurons had markedly decreased total dendritic length and the number of dendritic branches based on morphologic analysis (Fig. 6d, e). High power imaging analysis of dendritic spines showed reduced spine density, particularly thin type of spines, in these new-born DG neurons from Cre expressing mice, compared with that of controls (Fig. 6f-h). These results suggest that neogenin in NSCs/NPCs or immature neurons plays a role in promoting new-born neuronal dendritic morphogenesis and maturation.

We next tested this view in control and $\mathrm{Neo}^{\text {GliiCreER }}$ CKO mice, which were injected with tamoxifen at $\mathrm{P} 45$ for 1 month (Supplementary Fig. S6A). The tdTomato and NeuN double positive neurons (as new born DG neurons) were examined (Supplementary Fig. S6B). Again, dendritic complexity, length, and arborization were all

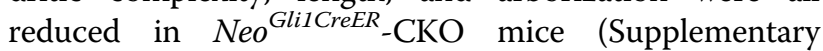
Fig. S6C-F). Similar to the results described in Fig. 6, the dendritic spine density and thin spines were largely decreased in neogenin depleted neurons (Supplementary Fig. S6G-I). These results thus confirmed neogenin's function in new born DG neuronal dendritic morphogenesis.

\section{Impairment of excitatory synaptic transmission and depressive-like behavior in mice that selectively knocked out neogenin in NSCs/NPCs}

We next asked whether loss of neogenin has functional deficit in their synaptic transmission. To this end, the retrovirus-expressing Cre was injected into hippocampus to infect NSCs at DG of $\mathrm{NeO}^{+/+} ; \mathrm{Ai} 9$ or $\mathrm{Ne}^{f / f} ; \mathrm{Ai} 9$ mice for 1 month; and new-born neurons expressing tdTomato were used for electrophysiological recording (Fig. 7a, b). As shown in Fig. 7c-e, the frequency, but not the amplitude, of miniature EPSCs (mEPSCs) was lower in Neo fff; $A i 9$ mice than that of control $\left(\mathrm{NeO}^{+/+} ; \mathrm{Ai} 9\right)$ mice. However, the frequency and amplitude of mIPSCs were comparable levels in the mutant neurons to that of control neurons (Fig. $7 \mathrm{f}-\mathrm{h}$ ). These results thus suggest that neogenin is essential for DG new born neuronal excitatory synaptic transmission.

Adult hippocampal neurogenesis is believed to be critical for prevention of depressive-like behavior and mood disorders ${ }^{40}$. Neogenin regulation of hippocampal neurogenesis thus leads us to speculate a role for neogenin in preventing depressive-like behavior. To test this speculation, we performed well-established mouse depressive- 
a

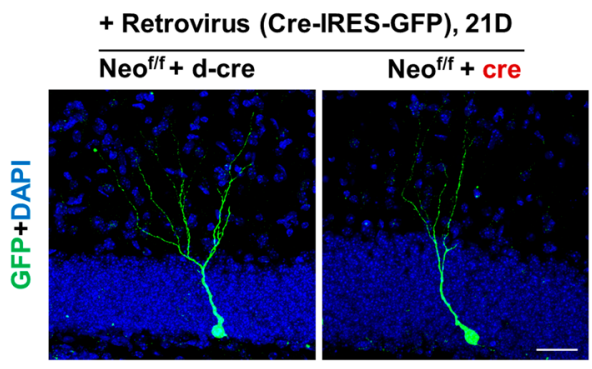

C

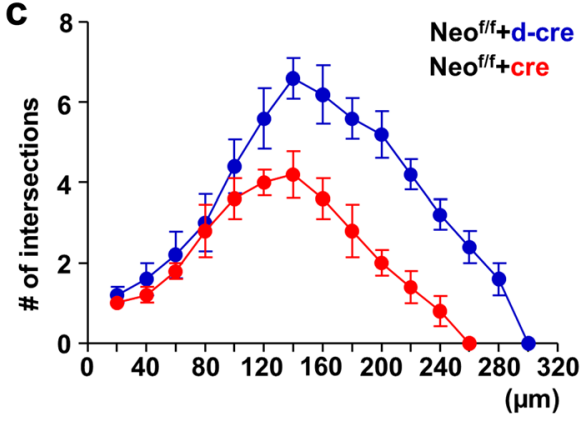

b

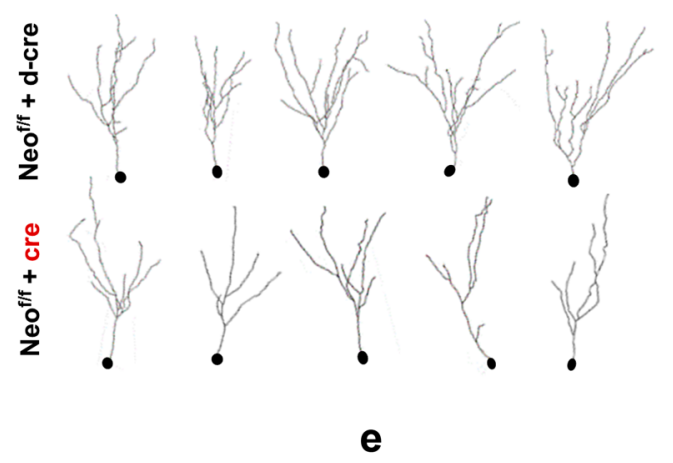

c

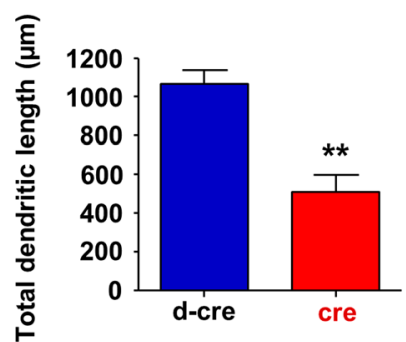

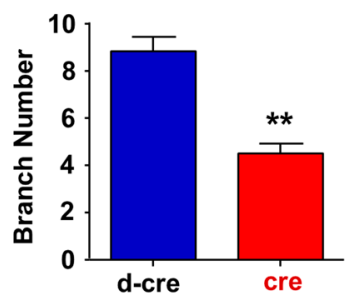

f

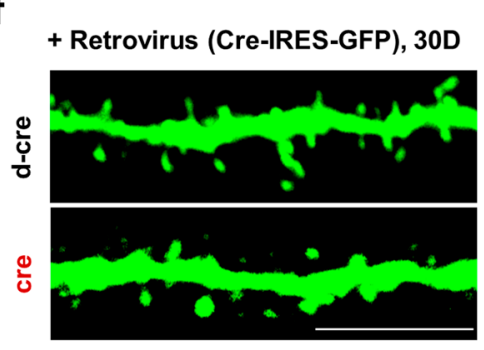

g

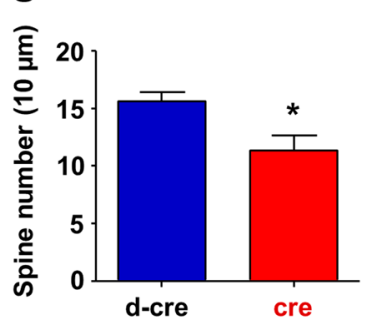

h

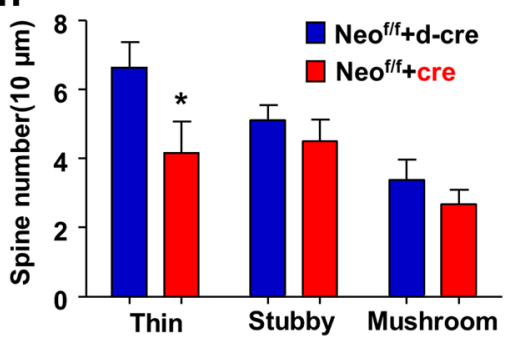

Fig. 6 Impaired dendritic morphogenesis in neogenin deficient new-born neurons. a Retroviruses expressing d-cre or cre were injected into DG of Neo f/f mice for 21 days. Scale bars $=20 \mu \mathrm{m}$. b Tracing of representative new-born neurons in the DG from both groups. $\mathbf{c}$ Sholl analysis of the dendritic complexity in $\mathbf{b}$ by ImageJ software. ( $n \geq 50$ cells). $\mathbf{d}$ Quantification of the dendritic total length. ( $n \geq 50$ cells). e Quantification of the branch number. $(n \geq 50$ cells). f Representative spine images of neurons infected with retrovirus expressing $d$-cre or cre for 1 -month. Scale bars $=5 \mu m$. $\mathbf{g}$ Quantitative analysis of the number of spines in $\mathrm{d}$-cre or cre expressing new-born neurons. $\mathbf{h}$ Quantitative analysis of different types of spines in $\mathrm{d}$-cre or cre expressing new-born neurons. Data are mean \pm SEM. ( $n \geq 30$ cells). ${ }^{*} P<0.05$; ${ }^{* *} P<0.01$. Student's $t$-test

like behavioral paradigms, including TST (tail suspension test), FST (forced swim test), and sucrose preference test. Both control $\left(\mathrm{NeO}^{+/+} ; \mathrm{Gli1}_{-\mathrm{CreER}}{ }^{T 2} ; \mathrm{Ai9}\right)$ and $\mathrm{Neo}^{\text {GliICreER }}$ CKO mice were injected with tamoxifen for 1 month

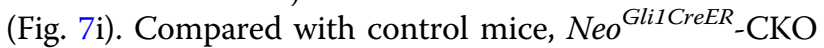
mice displayed longer immobility time in both TST and FST tests (Fig. 7j, k). For the sucrose preference test, the consumption ratio of sucrose to water was lower in Neo ${ }^{\text {GliICreER }}$-CKO mice than that of controls (Fig. 7l), indicating reduced preference for a palatable sucrose solution. These results suggest a depressive-like behavior

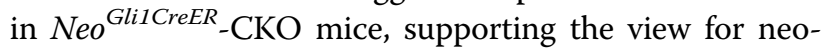
genin in adult hippocampal NSCs/NPCs to promote neurogenesis as well as to prevent depressive-like behavior.

\section{Discussion}

Here, we provide evidence for neogenin's functions in regulating adult mouse hippocampal neurogenesis, and propose a working model described in Fig. $7 \mathrm{~m}$. In this model, neogenin expression in NSCs/NPCs promotes their proliferation likely by increasing Gli1, a positive regulator of NSCs/NPCs proliferation ${ }^{41,42}$. In addition, neogenin in NSCs/NPCs prevents their astrocytic differentiation, but not neuronal differentiation. Furthermore, neogenin in immature neurons enhances dendritic outgrowth and branching, and thus elevating their excitatory neurotransmission and preventing depressive-like behavior. Taken together, these results not only identify neogenin's unrecognized functions in adult hippocampal neurogenesis, but also implicate 


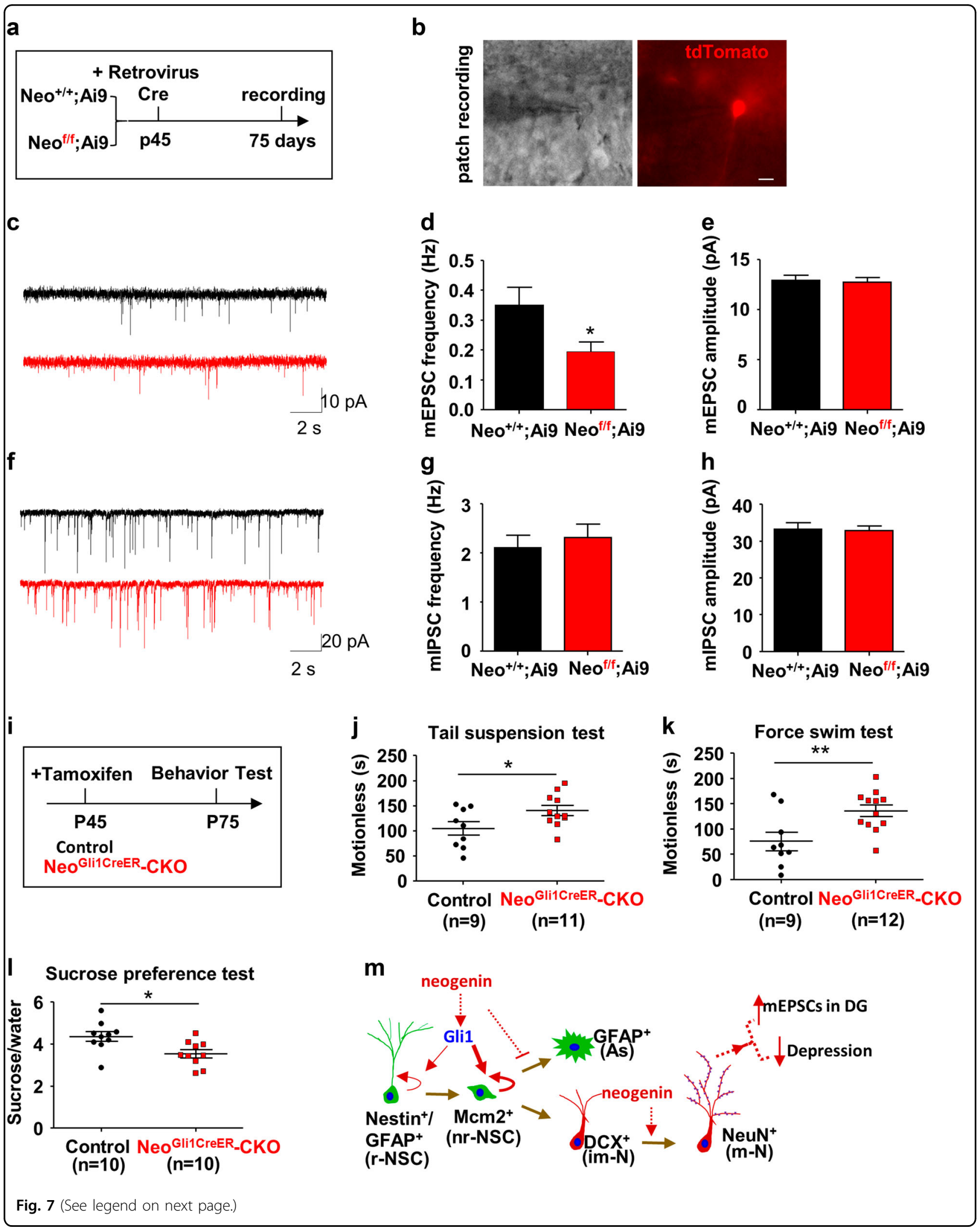


(see figure on previous page)

Fig. 7 Impairment of excitatory synaptic transmission and depressive-like behavior in mice that selectively knocked out neogenin in NSCs/NPCs. a Schematic drawing showed the strategy for electrophysiological recording. $\mathbf{b}$ Recording of tdTomato ${ }^{+}$DG new-born neurons (red) in both $\mathrm{NeO}^{+/+} ; \mathrm{Aig}$ (control) and Neo ${ }^{\mathrm{ff}} ; \mathrm{Aig}$ (knockout neogenin) mice, Scale bars $=10 \mu \mathrm{m}$. $\mathbf{c}$ Representative traces of mEPSC of new-born neurons from $\mathrm{NeO}^{+/+} ; \mathrm{Aig}$ and $\mathrm{NeO}^{f / f} ; \mathrm{Aig}$ mice. $\mathbf{d}, \mathbf{e}$ Quantitative analysis of the frequency $\mathbf{d}$ and amplitude $\mathbf{e}$ of mEPSC. ( $n \geq 10$ neurons). $\mathbf{f}$ Representative traces of

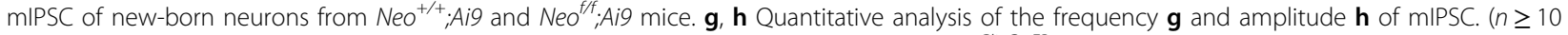
neurons). i Schematic diagram of tamoxifen treatment and behavior test. $\mathbf{j}$, $\mathbf{k}$ Control and Neo ${ }^{\text {Gli }}{ }^{\text {CreER }}$-CKO mice were subjected to the TST $\mathbf{j}$ and FST $\mathbf{k}$, and quantification of the immobility time in both groups. I Control and Neo ${ }^{\text {Gli CreER }}$-CKO mice were subjected to sucrose preference test. The ratio of sucrose verse water consumed was quantified. Data are presented as the mean \pm SEM. ( $n \geq 9$ per genotype). ${ }^{*} P<0.05 ;{ }^{* *} P<0.01$. Student's $t$-test. (m) Model of neogenin's functions in adult hippocampal neurogenesis

neogenin as a potential drug-able target for depression disorders.

Our results that neogenin is highly expressed in adult mouse hippocampal NSCs/NPCs in vivo and in vitro (Fig. 1) are in line with the reports for neogenin to be highly expressed in embryonic and adult $\mathrm{NSCs}^{32,43-46}$. Further supporting this view, neogenin is also detectable in $\mathrm{Nestin}^{+}$and Sox2 ${ }^{+}$hippocampal stem cells in rats ${ }^{47}$. It is found that neogenin regulates neuroblast migration and terminal differentiation in the $\mathrm{OB}^{26}$, and controls the balance of neurons and glial cells produced in the olfactory epithelium ${ }^{27}$. Whereas these reports demonstrate neogenin's functions in adult SVZ-OB neurogenesis and olfactory epithelium development, it remains unclear about neogenin's function in adult mouse hippocampal NSCs/NPCs. Here we addressed this issue by taking advantage of various neogenin conditional knock out mice

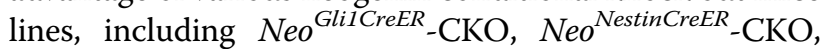
$\mathrm{Neo}{ }^{G_{F A P C r e E R}}$-CKO, and $\mathrm{NeO}^{\mathrm{NexCre}}$-CKO. When neogenin was selectively depleted in NSCs/NPCs (e.g., in Neo Gli1C${ }^{r e E R}{ }_{-} \mathrm{CKO}$ and Neo ${ }^{\text {NestinCreER}}-\mathrm{CKO}$ mice), NSCs/NPCs' proliferation and neurogenesis were impaired, but their astrogliogenesis was elevated (Figs. 2-5), demonstrating neogenin's function in regulating adult hippocampal neurogenesis.

Neogenin regulating adult hippocampal neurogenesis appears to have three different aspects. First, neogenin promotes NSCs/NPCs' proliferation, thus, increasing new born neurons. This is supported by our observations that the reduced tdTomato ${ }^{+}$cells or $\mathrm{DCX}^{+}$immature neurons in neogenin mutant DG cooperated well with the decreased proliferative NSCs/NPCs (Figs. 2, 4 and 5b), whereas the neural differentiation accessed in adult hippocampus and in NSCs culture were not affected by neogenin depletion (Figs. 3b, 5e). However, it remains unclear whether decreased proliferative NSCs/NPCs in neogenin mutant DG is due to the inhibition of proliferation speed or rate. Although more NSCs/NPCs were found to exit the cell cycle when neogenin was depleted (Fig. 4b, e), it is of great need to further explore the detailed functions of neurogenin in cell dividing. Second, neogenin suppresses astrocytic differentiation from NSCs/NPCs, thus, enhancing neurogenesis. This is based on our observations of elevated $\mathrm{GFAP}^{+}$astrocytes $\left(\mathrm{GFAP}^{+} ; \mathrm{Td}^{+} / \mathrm{Td}^{+}\right)$in neogenin mutant DG (Fig. 3f) and increased $\mathrm{GFAP}^{+}$astrocytes in neogenin mutant NSCs culture (Fig. 5d). Third, neogenin enhances new born neuronal maturation by increasing dendritic outgrowth and branching and new synapse formation. Supporting this view are observations that dendritic length, branching and spines were markedly reduced, as well as glutamatergic neuro-transmission was impaired in neogenin deficient new born neurons of DG (Figs. 6, 7c).

The defective NSCs/NPCs proliferation in neogenin mutant DG appeared to be due in large to an impaired Shh signaling to Gli1 (Fig. 5). Gli1 is a GLI-Kruppel family member and sensitive to Shh activity, and expressed in both quiescent stem cells and transient-amplifying cells, but not immature neurons ${ }^{35}$. It is reduced in neogenindeficient NSCs (Fig. 5h-j), and expression of Gli1 into neogenin-deficient NSCs could restore their proliferation (Fig. $5 k, 1)$. These results support the view for neogenin to promote shh signaling to Gli1, thus, regulating NSC/NPC proliferation and neurogenesis (Fig. $7 \mathrm{~m}$ ). This view is also in agreement with the reports that neogenin appears to be a direct Shh downstream regulator in neural precursor proliferation $^{48}$; and neogenin interacts with CDO (cell adhesion molecule-related/down regulated by oncogenes), a co-receptor for Shh to induce Shh signaling to Gli $^{49,50}$.

Notice that we have previously reported a role for neogenin to promote neocortical astrocytic differentiation ${ }^{32}$. To our surprise, neogenin's function in hippocampus appeared to be different/opposite from that in neocortex, where, it plays a negative role in hippocampal astrogliogenesis (Fig. 3f, 5d). These observations thus suggest a brain region-dependent function of neogenin in regulating astrocytic differentiation. Neogenin's differential effects on astrogliogenesis may be resulted from its differential regulations of different signaling pathways. In neocortex, neogenin is necessary for BMP induced signaling (SMAD1/ $5 / 8$ phosphorylation) and astrocytic differentiation ${ }^{32}$; whereas in adult hippocampus, neogenin appears to be required for shh signaling to Gli1. Thus, it is of interest to further investigate how neogenin in different brain regions participates into different signaling pathways. 
The continuous generation of new neurons in adult hippocampus plays a critical role during brain development, and new-born neurons represent a distinct population of cells with enhanced synaptic plasticity ${ }^{51}$. The new-born neuronal dendritic development and synaptic plasticity have been implicated in mood regulation, such as depression ${ }^{52-54}$. In light of these observations and considering neogenin's function in new-born neuronal maturation and function, we speculate a role for neogenin to regulate depressive-like behavior. Indeed, this appears to be the case. Neo ${ }^{\text {GlilCreER }}$-CKO mice not only exhibit defective new born neuronal maturation and function, but also show depressive-like behavior. However, the relevant circuitry and molecular mechanisms underlying dendritic development remain largely unknown, it is thus of great interest to further investigate neogenin's function in newborn neuron maturation and integration to the hippocampal circuits.

\section{Materials and methods}

\section{Animals and mouse breeding}

Neogenin mutant mice, generated by Bay Genomics, were kindly provided by Dr. Sue Ackerman (The Jackson Laboratory). Neogenin ${ }^{f / f}$ (Neo $\left.{ }^{f / f}\right)$ mice were maintained and genotyped as described previously ${ }^{32}$. Nestin-Cre, NexCre, Gli1-CreER ${ }^{T 2}$, Nestin-CreER ${ }^{T 2}, A i 9$ mice were purchased from the Jackson Laboratory. GFAP-CreER ${ }^{T 2}$ mice were a gift from K.D. McCarthy (University of North Carolina) ${ }^{55}$. We crossed Gli1-CreER ${ }^{T 2}$ mice with $A i 9$ mice to get Gli1-CreER ${ }^{T 2} ; A i 9$ mice (Supplementary Fig. S1A, B). $\mathrm{Neo}^{w / w}$;Gli1-CreER ${ }^{T 2} ; \mathrm{Ai} 9$ (Control) and $\mathrm{Neo}^{\mathrm{fff}}$;Gli1$\mathrm{CreER}^{T 2}$;Ai9 (Neo GliICreER $\left._{-} \mathrm{CKO}\right)$ mice were generated as described in Supplementary Fig. S2A, B. Neo ${ }^{\text {Nestin }}$-CKO,

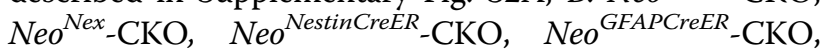
$\mathrm{Neo}^{f / f} ; \mathrm{Ai} 9$ mice were generated by crossing $\mathrm{Neo}{ }^{f / f}$ with Nestin-Cre, Nex-Cre, Nestin-CreER ${ }^{T 2}$, GFAP-CreER ${ }^{T 2}$ and $A i 9$ mice, respectively. The $A i 9$ mice contain a loxPflanked STOP cassette preventing transcription of a CAG promoter-driven red fluorescent protein variant (tdTomato). Thus, tdTomato is expressed following Cre mediated recombination. All the mouse lines indicated above were maintained in C57BL/6 strain background for $>6$ generations. To exclude the potential interference of different sex, only male mice were examined throughout the experiments. All mouse procedures used in this study were performed in accordance with protocols approved by the Institutional Animal Care and Use Committee at Augusta University.

\section{Antibody and plasmids}

Primary antibodies used in this project and their final concentrations were as follow: anti- $\beta$-gal (aves, 1:1000), anti-Nestin (Sigma, 1:300), anti-GFAP (Dako, 1:1000), anti-Mcm2 (BD, 1:500), anti-DCX (Santa Cruz, 1:200),
anti-NeuN (Millipore, 1:1000), anti-Brdu (Accurate chemical \& scientific corporation, 1:500), anti-Ki67 (Millipore, 1:200), anti-cleaved-caspase3 (CST, 1:200), anti-GFP (aves, 1:500), anti-neognin (Santa Cruz, 1:200), anti-Gli1 (abcam, 1:300), anti-GAPDH (Santa Cruz, 1:500). All the corresponding conjugated secondary antibody (1:1000) were purchased from Invitrogen. Nuclei were stained with 4',6-diamidino-2-phenylindole (DAPI) (1:1000, Roche). dCre and Cre plasmids were a kind gift from Dr. WeiXiang Guo in University of Chinese Academy of Sciences. pBABEPuro-HA-Gli1 (\#62967) plasmid was purchased from addgene.

\section{Drug treatment}

To inducible knockout neogenin in specific cell lineage in vivo, Control and Neo ${ }^{\text {GliICreER }}-\mathrm{CKO}$ mice; $\mathrm{Neo}^{f / f}$ and $\mathrm{Neo}^{\text {NestinCreER}}-\mathrm{CKO}$ mice; $\mathrm{Neo}^{f / f}$ and $\mathrm{NeO}^{\text {GFAPCreER}}-\mathrm{CKO}$ mice were given indicated injections of tamoxifen based on a published paradigm ${ }^{54}$. Tamoxifen (Sigma) was dissolved in corn oil at $20 \mathrm{mg} / \mathrm{ml}$. For Brdu incorporation assay, mice were given four injections of Brdu $(50 \mathrm{mg} / \mathrm{kg} /$ time, 1 time $/ 4 \mathrm{~h}$ ) within $12 \mathrm{~h}$ as previously described ${ }^{56}$. Mice were then sacrificed and perfused for Brdu immunostaining to analyze the proliferation index.

\section{X-gal staining}

$\mathrm{NeO}^{+/-}$mice were deeply anesthetized with ketamine/ xylazine (Sigma, 100/20 mg/kg, respectively, ip), and then perfused with $0.01 \mathrm{M}$ phosphate buffered saline (phosphate-buffered saline (PBS), $\mathrm{pH}=7.4$ ) followed by $4 \%$ paraformaldehyde (PFA) in PBS. The brains were sectioned into $40 \mu \mathrm{m}$-thick slices with a freezing microtome, and incubated with $\mathrm{X}$-gal staining solution for $12-16 \mathrm{~h}$ at $37^{\circ} \mathrm{C}$. After rinsed three times in PBS, the slices were counterstained with neutral red for $50 \mathrm{~s}$, washed twice with $\mathrm{dd}_{2} \mathrm{O}$, and over-laid with Permount TM Mounting Medium. Finally, the slices were covered with glass coverslips for imaging and analyze.

\section{Stereological cell counting}

For quantification of tdTomato ${ }^{+}$cells, immature neuron $\left(\mathrm{DCX}^{+}\right)$and proliferating $\mathrm{NSC} / \mathrm{NPC}\left(\mathrm{Brdu}^{+}\right.$or Ki $67^{+}$) densities, $40-\mu \mathrm{m}$ thick brain sections were immunostained with RFP, DCX, Brdu and Ki67 antibodies. One in every eight serial sections starting at the beginning of hippocampus (Bregma $-1.06 \mathrm{~mm}$ ) to the end of hippocampus (Bregma $-3.80 \mathrm{~mm}$ ) were examined with a Zeiss confocal system (FM300), and each section was separated into ten $z$-plane images by a $4-\mu \mathrm{m}$ step. About eight sections of each DG were counted, and total counts of eight examined sections were multiplied by 8 to estimate the total number of tdTomato ${ }^{+}$cells per DG. To quantify the cell density (number/volume) $\left(\mathrm{DCX}^{+}, \mathrm{Ki}^{+} 7^{+}\right.$ or $\mathrm{Brdu}^{+}$), we firstly measured the volume (area $\times$ 
thickness, $40 \mu \mathrm{m}$ ) of every ninth section through the entire DG with ImageJ software, and then calculated the cell number per $\mathrm{mm}^{3}$. Finally, data were presented as the mean of each counted section.

\section{Primary culture of adult hippocampal NSCs in vitro}

Neurospheres were prepared and maintained as described previously with slight modification ${ }^{57,58}$. Twomonth-old male mice ( $\mathrm{Neo}^{f / \mathrm{f}}$ and $\mathrm{Neo}^{\mathrm{Nestin}}$-CKO) were killed and then dissected DG to culture adult hippocampal NSCs. About 2 weeks later, neurospheres will be formed and then passaged for further study. All the measurements were carried out with the same number of passages.

\section{Histology}

Two to three-month-old mice were perfused with $4 \%$ $(\mathrm{w} / \mathrm{v})$ PFA in phosphate buffer (PBS) (pH 7.4), and the dissected brains were postfixed in $2 \%$ PFA at $4{ }^{\circ} \mathrm{C}$ overnight. Coronal sections $(40 \mu \mathrm{m})$ were washed 3 times with PBS (5 min each) and treated with blocking reagent $(10 \%$ Donkey Serum $+0.5 \%$ Triton $100 \times$ ) for $1 \mathrm{~h}$, then incubated overnight at $4{ }^{\circ} \mathrm{C}$ with the primary antibody. Sections were washed 3 times and incubated with corresponding conjugated secondary antibody for $2 \mathrm{~h}$. DAPI was used for nucleus counter staining.

\section{Western blot}

Cultured NSCs were lysed in cell lysis buffer to get protein samples. According to the protein weight, we prefer 10\% SDS-PAGE gel to separate proteins and then transfer them onto the nitrocellulose (NC) membrane. After electro-transfer, nitrocellulose membranes were blocked in western wash solution plus 5\% low fat dried milk for $1 \mathrm{~h}$ at room temperature. Relative primary antibodies were diluted in proper concentrations to incubate NC membrane overnight at $4{ }^{\circ} \mathrm{C}$. Membranes were washed and incubated for $1 \mathrm{~h}$ at room temperature with an appropriate horseradish-peroxidase-conjugated secondary antibody (1:5000, Thermo). For quantitative analysis, protein bands detected by ECL (Pierce, Rockford, IL) were scanned into pictures and analyzed using Image $\mathrm{J}$ software.

\section{qRT-PCR}

Total RNA was isolated from cultured NSCs with Trizol reagent and purified RNA $(5 \mu \mathrm{g})$ was used for cDNA synthesis using the RevertAid First Strand cDNA Synthesis Kit (Thermo Scientific). cDNA products were used for subsequent qRT-PCR $(20 \mu \mathrm{l})$ containing the SYBR GreenER qPCR SuperMix Universal (Invitrogen). We quantified gene expression using the follow primers: Gli1, forward: 5'CTCAAACTGCCCAGCTTAACCC3'; reverse: 5'TGCGGCTGACTGTGTAAGCAGA3'; Gli2, forward:
5'ACACTGTGGAGGACTGCCTACA3'; reverse: 5'GGC ATCTCCATGCCACTGTCAT3'; Gli3, forward: 5'TCCA TGGCTCTCTACCACATC3'; reverse: 5'GTGGCAGCT GAGGGAAGGAT3'; ptc1, forward: 5'AACAAAAATT CAACCAAACCTC 3 ; r reverse: 5'TGTCTTCATTCCAGT TGATGTG3'; smo, forward: 5'TGCCACCAGAAGAACAAGCCA3'; reverse: 5'GCCTCCATTAGGTTAGTG CGG3'; neogenin, forward: 5'GAGATGGGGGACTCTA CCG3'; reverse: 5'TGTCAAGCACTTCTTCGTT3'; GAP DH, forward: 5'CATCACTGCCACCCAGAAGACTG3'; reverse: 5'ATGCCAGTGAGCTTCCCGTTCAG3'. All samples were amplified in duplicate, and every experiment was repeated at least independently 3 times. The amount of amplified target cDNA was converted using the $2^{-\Delta \Delta \mathrm{Ct}}$ method against the GAPDH to represent the relative levels in each sample.

\section{Production of retrovirus and virus grafting in vivo}

Production of retrovirus was performed as described previously with slight modification ${ }^{59}$. Briefly, retroviral plasmids were transfected into cultured 293-GPG cells using polyethyleneimine, 293-GPG cells are stably transfected packaging cells, retrovirus was collected at 48, 72, and $96 \mathrm{~h}$ post transfection, filtered by a $0.2 \mu \mathrm{m}$ filter, and then concentrated at 20,000 $\mathrm{rcf}$ for $2 \mathrm{~h}$ at $4{ }^{\circ} \mathrm{C}$ using a SW27 rotor (Beckman). Removed the supernant and resuspended the retrovirus in $100 \mu \mathrm{l}$ PBS.

For in vivo retrovirus grafting, 2-month old mice were anesthetized with ketamine/xylazine (Sigma, 100/20 mg/ $\mathrm{kg}$, respectively, ip), $1 \mu \mathrm{ld}$-Cre (an inactive Cre, as a control) retrovirus was injected into the left DG of brain and $1 \mu \mathrm{l}$ Cre retrovirus was injected into the other side. The injected coordinates were relative to bregma, caudal: $-2.0 \mathrm{~mm}$; lateral: $+/-1.6 \mathrm{~mm}$; ventral: $-2.0 \mathrm{~mm}$.

\section{Retrovirus infection in cultured NSCs in vitro}

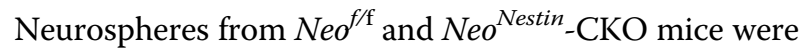
digested into single cells and seeded at $1 \times 10^{5}$ cells $/ \mathrm{ml}$. Subsequently, concentrated Gli1-HA retroviruses were added into proliferation medium to infect NSCs of Neo${ }_{\text {Nestin }}$-CKO mice. 3 days later, immunostaining of HA tag indicated the expressing efficiency of Gli1 in cultured NSCs in vitro.

\section{Electrophysiological recording}

Hippocampal slices were prepared as described previously $^{60}$. mEPSCs were recorded at holding potential of $-70 \mathrm{mV}$ in the presence of $20 \mu \mathrm{M}$ BMI and $1 \mu \mathrm{M}$ TTX, with the pipette solution containing (in $\mathrm{mM}$ ): $125 \mathrm{~K}$ gluconate, $5 \mathrm{KCl}, 10$ Hepes, 0.2 EGTA, $1 \mathrm{MgCl} 2,4 \mathrm{Mg}$ ATP, $0.3 \mathrm{Na}-\mathrm{GTP}$ and 10 phosphocreatine (pH 7.40, 285 mOsm). To record miniature IPSCs (mIPSCs), neurons were held at $-70 \mathrm{mV}$ in the presence of $20 \mu \mathrm{M}$ CNQX, $100 \mu \mathrm{M}$ DL-AP5 and $1 \mu \mathrm{M}$ TTX. Recording pipettes were 
filled with the solution contained (in $\mathrm{mM}$ ): $140 \mathrm{CsCl}, 10$ Hepes, 0.2 EGTA, $1 \mathrm{MgCl}_{2}$, $4 \mathrm{Mg}$-ATP, $0.3 \mathrm{Na}$-GTP, 10 phosphocreatine and 5 QX314 (pH 7.4, 285 mOsm). In all experiments, series resistance was controlled below 20 $\mathrm{M} \Omega$ and not compensated. Cells would be rejected if membrane potentials were more positive than $-60 \mathrm{mV}$; or if series resistance fluctuated more than $20 \%$ of initial values. All recordings were done at $32-34{ }^{\circ} \mathrm{C}$. Data were filtered at $1 \mathrm{kHz}$ and sampled at $10 \mathrm{kHz}$.

\section{Behavioral analysis}

Two to three-month old mice (male) were housed together and used for behavioral studies. The TST and FST were performed as described previously with slight modification $^{61,62}$. The last 4-min of a 6-min test were analyzed and the immobility time was measured directly. The sucrose preference test was carried out using a two bottle choice procedure. Single housed mice were habituated to drink $2 \%$ (wt/vol) sucrose solution (dissolved in water) for 3 days, then mice were given access to the two preweighed bottles, one containing water and the other containing $2 \%$ sucrose solution. We changed bottle positions every day and assessed water and sucrose solution consumption daily for 4 days. The consuming ratio of sucrose over water was used for measuring the sucrose preference.

\section{Statistical analysis}

All results presented in this study were from at least three independent experiments. Statistical analyses were performed using Student's $t$ test, or one-way ANOVA followed by a Bonferroni post hoc analysis. All data were presented as mean \pm SEM and described in the figure legends. A value of $P<0.05$ was considered to be significant. All graphics were prepared using GraphPad Prism 5 software.

\section{Acknowledgements}

This study was supported by grants from the National Institutes of Health [AG045781 and AG051773 to WCX]; US Department of Veterans Affairs [to WCX] and China scholarship council (201506620038). We thank Kim, Jimok (Augusta University) for technical support on electrophysiological recording. We thank members in Drs. Xiong and Mei laboratories for helpful discussions and suggestions.

\footnotetext{
Author details

${ }^{1}$ Key Laboratory of Molecular Epigenetics of Ministry of Education, Institute of Cytology and Genetics, Northeast Normal University, Changchun, Jilin 130024 China. ${ }^{2}$ Department of Neuroscience \& Regenerative Medicine and Department of Neurology, Augusta University, Augusta, GA 30912, USA. ${ }^{3}$ Department of Neurology, The affiliated hospital of Xuzhou Medical University, Xuzhou, Jiangsu Province 221002, China
}

\section{Author contributions}

W.C.X., L.M. and X.J.Z. designed the experiments. D.S., X.D.S., L.Z., D.H.L. and J.X. H. preformed the experiments. F.L.T. and J.X.P. contributed reagents/materials/ analysis. W.C.X. wrote the main manuscript text. All authors reviewed the manuscript.

\section{Competing interests}

The authors declare that they have no competing financial interests.

\section{Supplementary information}

The online version of this article (https://doi.org/10.1038/s41419-017-0019-2) contains supplementary material.

Received: 23 June 2017 Revised: 19 September 2017 Accepted: 2 October 2017

Published online: 08 January 2018

\section{References}

1. Mu, Y., Lee, S. W. \& Gage, F. H. Signaling in adult neurogenesis. Curr. Opin. Neurobiol. 20, 416-423 (2010).

2. Magavi, S. S., Mitchell, B. D., Szentirmai, O., Carter, B. S. \& Macklis, J. D. Adultborn and preexisting olfactory granule neurons undergo distinct experiencedependent modifications of their olfactory responses in vivo. J. Neurosci. 25, 10729-10739 (2005).

3. Lois, C., Garcia-Verdugo, J. M. \& Alvarez-Buylla, A. Chain migration of neuronal precursors. Science 271, 978-981 (1996).

4. Kuhn, H. G., Dickinson-Anson, H. \& Gage, F. H. Neurogenesis in the dentate gyrus of the adult rat: age-related decrease of neuronal progenitor proliferation. J. Neurosci. 16, 2027-2033 (1996).

5. Suh, $H$. et al. In vivo fate analysis reveals the multipotent and self-renewal capacities of Sox2+neural stem cells in the adult hippocampus. Cell Stem Cell 1, 515-528 (2007)

6. Lledo, P. M., Alonso, M. \& Grubb, M. S. Adult neurogenesis and functional plasticity in neuronal circuits. Nat. Rev. Neurosci. 7, 179-193 (2006).

7. Massa, F. et al. Conditional reduction of adult neurogenesis impairs bidirectional hippocampal synaptic plasticity. Proc. Natl. Acad. Sci. USA 108, 6644-6649 (2011).

8. Tang, M. M., Lin, W. J., Pan, Y. Q., Guan, X. T. \& Li, Y. C. Hippocampal neurogenesis dysfunction linked to depressive-like behaviors in a neuroinflammation induced model of depression. Physiol. Behav. 161, 166-173 (2016).

9. Valente, M. M. et al. alpha2delta ligands act as positive modulators of adult hippocampal neurogenesis and prevent depression-like behavior induced by chronic restraint stress. Mol. Pharmacol. 82, 271-280 (2012).

10. Shi, Y. et al. Expression and function of orphan nuclear receptor $T L X$ in adult neural stem cells. Nature 427, 78-83 (2004).

11. Ferri, A. L. et al. Sox2 deficiency causes neurodegeneration and impaired neurogenesis in the adult mouse brain. Development 131, 3805-3819 (2004).

12. Cheng, L. C., Pastrana, E., Tavazoie, M. \& Doetsch, F. miR-124 regulates adult neurogenesis in the subventricular zone stem cell niche. Nat. Neurosci. 12, 399-408 (2009).

13. Lai, K., Kaspar, B. K., Gage, F. H. \& Schaffer, D. V. Sonic hedgehog regulates adult neural progenitor proliferation in vitro and in vivo. Nat. Neurosci. 6, 21-27 (2003).

14. Lie, D. C. et al. Wnt signalling regulates adult hippocampal neurogenesis. Nature 437, 1370-1375 (2005).

15. Imayoshi, I., Sakamoto, M., Yamaguchi, M., Mori, K. \& Kageyama, R. Essential roles of Notch signaling in maintenance of neural stem cells in developing and adult brains. J. Neurosci. 30, 3489-3498 (2010).

16. Houser, C. R. Interneurons of the dentate gyrus: an overview of cell types, terminal fields and neurochemical identity. Prog. Brain Res. 163, 217-232 (2007).

17. Cameron, H. A., McEwen, B. S. \& Gould, E. Regulation of adult neurogenesis by excitatory input and NMDA receptor activation in the dentate gyrus. J. Neurosci. 15, 4687-4692 (1995)

18. Ge, S., Pradhan, D. A., Ming, G. L. \& Song, H. GABA sets the tempo for activitydependent adult neurogenesis. Trends Neurosci. 30, 1-8 (2007).

19. McAvoy, K. M. et al. Modulating neuronal competition dynamics in the dentate gyrus to rejuvenate aging memory circuits. Neuron 91, 1356-1373 (2016).

20. Wilson, N. H. \& Key, B. Neogenin interacts with RGMa and netrin-1 to guide axons within the embryonic vertebrate forebrain. Dev. Biol. 296, 485-498 (2006).

21. Rajagopalan, S. et al. Neogenin mediates the action of repulsive guidance molecule. Nat. Cell Biol. 6, 756-762 (2004). 
22. Zhang, A. S., Yang, F., Wang, J., Tsukamoto, H. \& Enns, C. A. Hemojuvelinneogenin interaction is required for bone morphogenic protein-4-induced hepcidin expression. J. Biol. Chem. 284, 22580-22589 (2009).

23. Matsunaga, E., Nakamura, H. \& Chedotal, A. Repulsive guidance molecule plays multiple roles in neuronal differentiation and axon guidance. J. Neurosci. 26, 6082-6088 (2006).

24. Mawdsley, D. J. et al. The Netrin receptor Neogenin is required for neural tube formation and somitogenesis in zebrafish. Dev. Biol. 269, 302-315 (2004).

25. Matsunaga, E. et al. RGM and its receptor neogenin regulate neuronal survival. Nat. Cell Biol. 6, 749-755 (2004).

26. O'Leary, C. J. et al. The Netrin/RGM receptor, Neogenin, controls adult neurogenesis by promoting neuroblast migration and cell cycle exit. Stem Cells $\mathbf{3 3}$, 503-514 (2015)

27. Kam, J. W. et al. RGMB and neogenin control cell differentiation in the developing olfactory epithelium. Development 143, 1534-1546 (2016).

28. Zhou, Z. et al. Neogenin regulation of BMP-induced canonical Smad signaling and endochondral bone formation. Dev. Cell 19, 90-102 (2010).

29. Hong, M., Schachter, K. A., Jiang, G. \& Krauss, R. S. Neogenin regulates Sonic Hedgehog pathway activity during digit patterning. Dev. Dyn. 241, 627-637 (2012).

30. Kuns-Hashimoto, R., Kuninger, D., Nili, M. \& Rotwein, P. Selective binding of RGMc/hemojuvelin, a key protein in systemic iron metabolism, to BMP-2 and neogenin. Am. J. Physiol. Cell Physiol. 294, C994-C1003 (2008).

31. Lee, D. H. et al. Neogenin inhibits HJV secretion and regulates BMP-induced hepcidin expression and iron homeostasis. Blood 115, 3136-3145 (2010).

32. Huang, Z. et al. Neogenin promotes BMP2 activation of YAP and Smad1 and enhances astrocytic differentiation in developing mouse neocortex. J. Neurosci. 36, 5833-5849 (2016).

33. Mitchell, K. J. et al. Functional analysis of secreted and transmembrane proteins critical to mouse development. Nat. Genet. 28, 241-249 (2001).

34. Machold, R. et al. Sonic hedgehog is required for progenitor cell maintenance in telencephalic stem cell niches. Neuron 39, 937-950 (2003).

35. Ahn, S. \& Joyner, A. L. In vivo analysis of quiescent adult neural stem cells responding to Sonic hedgehog. Nature 437, 894-897 (2005).

36. Palma, V. et al. Sonic hedgehog controls stem cell behavior in the postnatal and adult brain. Development 132, 335-344 (2005).

37. Kempermann, G., Jessberger, S., Steiner, B. \& Kronenberg, G. Milestones of neuronal development in the adult hippocampus. Trends Neurosci. 27, 447-452 (2004).

38. Bonaguidi, M. A. et al. In vivo clonal analysis reveals self-renewing and multipotent adult neural stem cell characteristics. Cell 145, 1142-1155 (2011).

39. Smrt, R. D. et al. MicroRNA miR-137 regulates neuronal maturation by targeting ubiquitin ligase mind bomb-1. Stem Cells 28, 1060-1070 (2010).

40. Kempermann, G. \& Kronenberg, G. Depressed new neurons--adult hippocampal neurogenesis and a cellular plasticity hypothesis of major depression. Biol. Psychiatry 54, 499-503 (2003).

41. Merchant, A., Joseph, G., Wang, Q., Brennan, S. \& Matsui, W. Gli1 regulates the proliferation and differentiation of HSCs and myeloid progenitors. Blood $\mathbf{1 1 5}$ 2391-2396 (2010).

42. Breunig, J. J. et al. Primary cilia regulate hippocampal neurogenesis by mediating sonic hedgehog signaling. Proc. Natl. Acad. Sci. USA 105, 13127-13132 (2008).
43. Gad, J. M., Keeling, S. L., Wilks, A. F., Tan, S. S. \& Cooper, H. M. The expression patterns of guidance receptors, DCC and Neogenin, are spatially and temporally distinct throughout mouse embryogenesis. Dev. Biol. 192, 258-273 (1997).

44. Fitzgerald, D. P., Bradford, D. \& Cooper, H. M. Neogenin is expressed on neurogenic and gliogenic progenitors in the embryonic and adult central nervous system. Gene Expr. Patterns 7, 784-792 (2007).

45. Bradford, D., Faull, R. L., Curtis, M. A. \& Cooper, H. M. Characterization of the netrin/RGMa receptor neogenin in neurogenic regions of the mouse and human adult forebrain. J. Comp. Neurol. 518, 3237-3253 (2010).

46. van den Heuvel, D. M., Hellemons, A. J. \& Pasterkamp, R. J. Spatiotemporal expression of repulsive guidance molecules (RGMs) and their receptor neogenin in the mouse brain. PLoS One 8, e55828 (2013).

47. Hong, N., Kim, M. H., Min, C. K., Kim, H. J. \& Lee, J. H. The co-expression of Neogenin with SOX2 in hippocampal neurons. Biochem. Biophys. Res. Commun. 490, 453-459 (2017).

48. Milla, L. A. et al. Neogenin1 is a Sonic Hedgehog target in medulloblastoma and is necessary for cell cycle progression. Int. J. Cancer 134, 21-31 (2014).

49. Kang, J. S. et al. Netrins and neogenin promote myotube formation. J. Cell Biol. 167, 493-504 (2004).

50. Kwon, Y. R. et al. The Shh coreceptor Cdo is required for differentiation of midbrain dopaminergic neurons. Stem Cell Res. 13, 262-274 (2014).

51. Ge, S., Yang, C. H., Hsu, K. S., Ming, G. L. \& Song, H. A critical period for enhanced synaptic plasticity in newly generated neurons of the adult brain. Neuron 54, 559-566 (2007).

52. Zheng, C. \& Zhang, T. Synaptic plasticity-related neural oscillations on hippocampus-prefrontal cortex pathway in depression. Neuroscience $\mathbf{2 9 2}$ 170-180 (2015).

53. Christie, B. R., Kerr, D. S. \& Abraham, W. C. Flip side of synaptic plasticity: longterm depression mechanisms in the hippocampus. Hippocampus 4, 127-135 (1994).

54. Li, Y., Li, Y., McKay, R. M., Riethmacher, D. \& Parada, L. F. Neurofibromin modulates adult hippocampal neurogenesis and behavioral effects of antidepressants. J. Neurosci. 32, 3529-3539 (2012).

55. Casper, K. B., Jones, K. \& McCarthy, K. D. Characterization of astrocyte-specific conditional knockouts. Genesis 45, 292-299 (2007).

56. Hayes, N. L. \& Nowakowski, R. S. Dynamics of cell proliferation in the adult dentate gyrus of two inbred strains of mice. Brain Res. Dev. Brain Res. 134, 77-85 (2002).

57. Guo, W., Patzlaff, N. E., Jobe, E. M. \& Zhao, X. Isolation of multipotent neural stem or progenitor cells from both the dentate gyrus and subventricular zone of a single adult mouse. Nat. Protoc. 7, 2005-2012 (2012).

58. Sun, D. et al. Regulation of neural stem cell proliferation and differentiation by Kinesin family member 2a. PLoS One 12, e0179047 (2017).

59. Smrt, R. D. et al. Mecp2 deficiency leads to delayed maturation and altered gene expression in hippocampal neurons. Neurobiol. Dis. 27, 77-89 (2007).

60. Sun, X. D. et al. Lrp4 in astrocytes modulates glutamatergic transmission. Nat Neurosci. 19, 1010-1018 (2016).

61. Can, A. et al. The tail suspension test. J. Vis.1 Exp. 59, e3769 (2012).

62. Can, A. et al. The mouse forced swim test. J. Vis. Exp. 59, e3638 (2012). 